
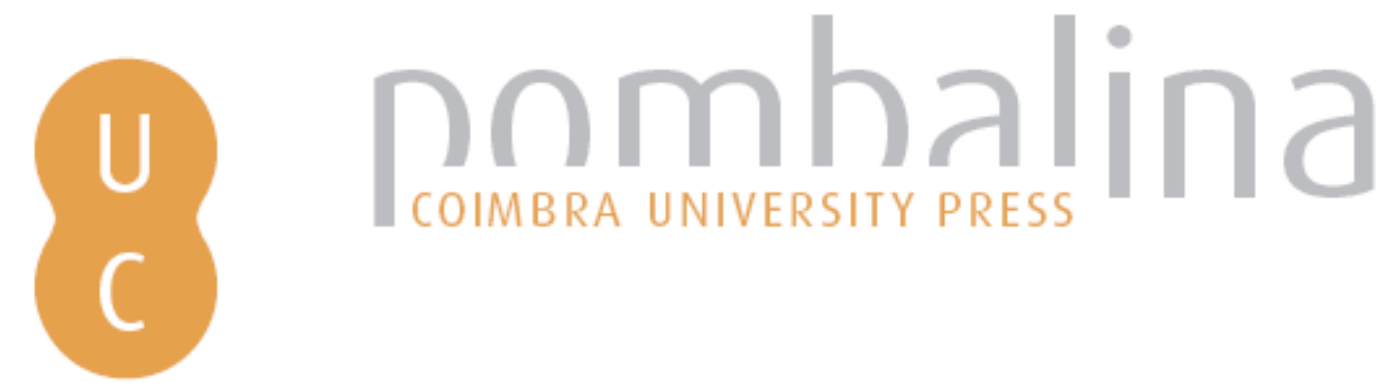

\title{
Perfil de la investigación y de los investigadores que se han formado en el Programa Doctoral de Documentación en España: El caso cel Departamento de Biblioteconomía y Documentación de la Universidad Carlos III de Madrid (Uc3m)
}

Autor(es): Ortiz-Sánchez, Sarivette; Martín-Moreno, Carmen

Publicado por: Imprensa da Universidade de Coimbra

URL persistente:

URI:http://hdl.handle.net/10316.2/31963

DOI:

DOI:http://dx.doi.org/10.14195/978-989-26-0319-3_39

Accessed : $\quad$ 26-Apr-2023 15:21:32

A navegação consulta e descarregamento dos títulos inseridos nas Bibliotecas Digitais UC Digitalis, UC Pombalina e UC Impactum, pressupõem a aceitação plena e sem reservas dos Termos e Condições de Uso destas Bibliotecas Digitais, disponíveis em https://digitalis.uc.pt/pt-pt/termos.

Conforme exposto nos referidos Termos e Condições de Uso, o descarregamento de títulos de acesso restrito requer uma licença válida de autorização devendo o utilizador aceder ao(s) documento(s) a partir de um endereço de IP da instituição detentora da supramencionada licença.

Ao utilizador é apenas permitido o descarregamento para uso pessoal, pelo que o emprego do(s) título(s) descarregado(s) para outro fim, designadamente comercial, carece de autorização do respetivo autor ou editor da obra.

Na medida em que todas as obras da UC Digitalis se encontram protegidas pelo Código do Direito de Autor e Direitos Conexos e demais legislação aplicável, toda a cópia, parcial ou total, deste documento, nos casos em que é legalmente admitida, deverá conter ou fazer-se acompanhar por este aviso.

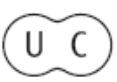


Maria Manuel Borges

Elias Sanz Casado

Coordenação

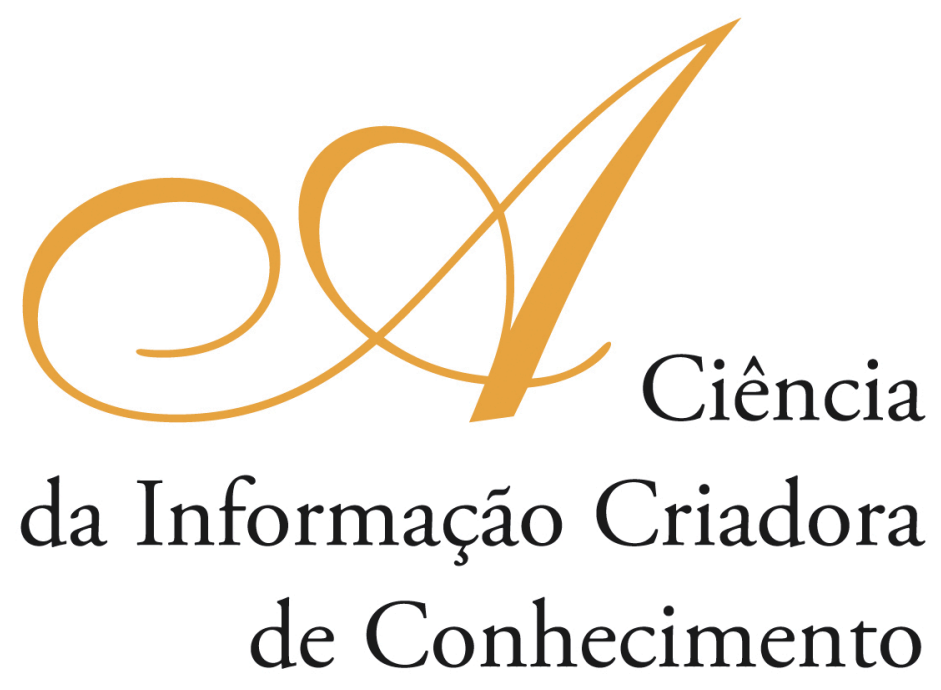

Vol. I

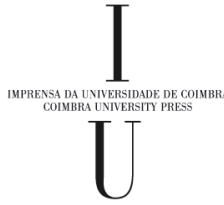

- COIMBRA 2009 


\author{
Perfil de la Investigación y de los Investigadores \\ Que se han formado en el Programa Doctoral \\ de Documentación en España: El caso cel Departamento \\ de Biblioteconomía y Documentación de la Universidad Carlos III de \\ MAdrid (UC3M)
}

\author{
Sarivette Ortiz-Sánchez \\ Universidad Carlos III de Madrid (Espanha) \\ Carmen Martín-Moreno \\ Universidad Carlos III de Madrid (Espanha)
}

\title{
Resumen
}

Para conocer el impacto de los cambios en normativas universitarias es importante conocer el perfil de sus departamentos. Los estudios bibliométricos han demostrado ser una alternativa para identificar estos perfiles. Las tesis doctorales por su parte, permiten conocer la producción de un departamento y su capacidad para formar investigadores. Con este trabajo se buscó identificar el perfil de la producción y de los investigadores formados en un programa doctoral en documentación mediante el estudio de caso de la Universidad Carlos III (UC3M). Nos centramos en unos objetivos específicos que buscaban conocer el perfil de la producción en tesis del mencionado departamento, su visibilidad en el extranjero y el perfil actual de los doctores formados en el mismo. Nos basamos en la información obtenida de la Oficina de Tercer Ciclo y en el catálogo de la biblioteca de la UC3M para identificar el perfil de las tesis. Se distribuyó un cuestionario entre los autores de las tesis que nos permitió identificar su perfil actual. Se observó que el Departamento de Documentación de la UC3M defiende un promedio de 4,14 tesis por año. Por otra parte la procedencia de estudiantes matriculados en el doctorado muestra la visibilidad que tiene esta universidad en América. En cuento a la colaboración en las tesis se percibe que han recibido participación tanto de América como de Europa. Destacan Brasil, México y Puerto Rico ya que la UC3M no solo ha formado doctores para estos países sino que también ha recibido colaboraciones de doctores procedentes de los mismos países. El perfil actual de los doctores formados en esta universidad los coloca principalmente en el ambiente académico y continúan trabajando con su tema de tesis aunque lo han enriquecido con temas nuevos. Estos prefieren las revistas tanto para publicar como para mantenerse actualizados en sus temas de investigación.

\begin{abstract}
In order to understand the impact of changes in university norms, it is important to know the profile of its departments. The bibliometric studies have demonstrated to be an alternative to identify these profiles. The doctoral theses allow to know the production of a department and its capability to form investigators. This work looked for to identify the profile of the production and the investigators formed in a doctoral program in library science by using the case study of the University Carlos III (UC3M). We were centered in specific objectives
\end{abstract}


to know the profile of the production in theses of the mentioned department, to know its visibility and to know the present profile of the formed doctors. The information was based on obtained data from the Office of Third Cycle and the catalogue of the library of the UC3M to identify the profile of theses. A questionnaire was distributed between the authors of the theses that allowed us to identify their present profile. With these instruments, it was observed that the Department of Documentation of the UC3M produce an average of 4.14 theses per year. On the other hand, the origin of students registered in the doctorate program shows the visibility that has this university in America. In terms of the collaboration with the theses it is perceived that they have received as much participation from America as from Europe. The present profile of the doctors formed in this university mainly places them in the academic atmosphere and continue working with its thesis subject although they have enriched it with new subjects. They prefer the journals not only to publish their work but also to stay updated in their subjects of investigation.

\section{Introducción}

La normativa relacionada con el ámbito educativo universitario ha ido cambiando para adaptarse a las necesidades y evolución de la sociedad que les rodea. Para conocer el impacto real de estos cambios en un departamento universitario es importante conocer el perfil del mismo antes y después de la implementación de dichas modificaciones. De acuerdo con Sanz Casado \& Martín Moreno (1997) los estudios bibliométricos han desarrollado técnicas que permiten conocer diferentes características de los usuarios, entre ellas su producción científica. En este sentido, los estudios bibliométricos son una alternativa para realizar los perfiles antes mencionados, de manera que los departamentos puedan conocer su producción investigadora y la visibilidad de estos esfuerzos antes y después de realizar cambios educativos y estructurales.

Las tesis doctorales son el resultado de una investigación realizada por un aspirante a doctor que ha completado una preparación académica en particular. Esto convierte dichas tesis en un buen instrumento para observar, no solo la producción investigadora de un departamento universitario, sino también su capacidad para formar investigadores. En España se han realizado varios estudios relacionados con análisis de tesis, sin embargo, son pocos los que hacen un seguimiento del perfil investigador tanto de los autores de las mismas, como de la investigación realizada, un tipo de análisis que permite conocer el perfil de los investigadores formados como doctores por los departamentos universitarios.

En este trabajo se hace un estudio de caso sobre la trayectoria del Programa Oficial de Postgrado impartido por el Departamento de Biblioteconomía y Documentación de la Universidad Carlos III de Madrid (UC3M), a través de las tesis defendidas a partir de la puesta en marcha del Programa de Doctorado. Para ello, se presenta el perfil de las tesis defendidas desde el inicio del Programa hasta el año académico 2007-2008. Por tratarse de un estudio de caso, creemos que es interesante referirnos brevemente a la historia de dicho Departamento, así como de su programa doctoral.

Este departamento comenzó a impartir los cursos de Diplomatura en biblioteconomía y documentación en 1991 continuando con la Licenciatura en 1994, ambas en el Campus de Getafe, si bien en los 5 años siguientes extendió su oferta al campus de Colmenarejo. Originalmente la oferta en ambos campus fue para la modalidad 
presencial (Universidad Carlos III, 2009). Esta situación se mantuvo hasta 2007 en que se cambió la modalidad de enseñanza en el campus de Colmenarejo, y pasó a ser semi-presencial, añadiéndose a las alternativas presenciales de Getafe. A partir del año académico 2008-2009, este Departamento tiene presencia en todas las titulaciones que se imparten en la Universidad, mediante el curso transversal: Técnicas de búsqueda y uso de la información.

El Programa de doctorado por su parte comenzó en 1994, y siempre ha requerido de un período de formación seguido de otro de investigación conducente a la realización de una tesis doctoral. Desde sus comienzos ha sufrido diferentes cambios en función de las distintas regulaciones determinadas por el Ministerio de Educación, si bien las principales se han referido al número de créditos que debían cursarse en el periodo de formación. El último Real Decreto que regula estos estudios es de 2007. Con él, no ha cambiado la estructura del programa, pero permite culminar el periodo de formación con la obtención de un título de Máster. A este periodo le sigue el de investigación, necesario para la realización de la tesis doctoral, y que culminará al alcanzar el Grado de Doctor. A lo largo del tiempo transcurrido desde que comenzó el Programa de doctorado ha cambiado su nombre, y actualmente, los dos periodos del programa se conocen como Máster en Investigación en Documentación y Doctorado en Archivos y Bibliotecas en el Entorno Digital.

En 2004 estos estudios obtuvieron la Mención de Calidad del Ministerio de Educación y Ciencia, mención que conservan al día de hoy, dado que en el año académico 2008-2009 la Agencia Nacional de Evaluación de Calidad y Acreditación (ANECA) renovó la Mención de Calidad al programa de doctorado.

Una vez señalados los pasos que ha seguido el Departamento en la implantación de los distintos estudios que imparte, pasaremos a los objetivos planteados para realizar este trabajo. Estos han sido los siguientes

1. Identificar el perfil de la producción y del investigador como resultado de la formación recibida en un programa de doctorado español específico, el impartido por el Departamento de Biblioteconomía y Documentación de la UC3M.

2. Tratar de identificar la visibilidad del Departamento de Biblioteconomía y Documentación de la UC3M mediante la participación de instituciones extranjeras en los procesos de producción y evaluación de las tesis.

3. Conocer el perfil actual de los investigadores que se formaron en el Departamento de Biblioteconomía y Documentación de la Universidad Carlos III de Madrid y la continuidad que dio a su tema de tesis.

\section{Metodología}

Este trabajo se ha realizado con las 58 tesis leídas en el Departamento de Biblioteconomía y Documentación de la Universidad Carlos III a lo largo del periodo 1997-2008.

Para determinar las tesis defendidas, en primer lugar se realizó una búsqueda en el catalogo de la UC3M, que incluye los recursos disponibles en todas sus bibliotecas independientemente del campus. Dado que las tesis producidas en el Programa de Doctorado del Departamento de Biblioteconomía y Documentación, son asignadas 
a la biblioteca de Humanidades, Comunicación y Documentación, la búsqueda se limitó a dicha biblioteca, y el campo de MATERIAL se limitó a tesis. El resto de los campos de búsqueda de dicho catálogo se dejaron en blanco o con la alternativa de CUALQUIERA. Con esta estrategia se recuperaron todas las tesis disponibles en la mencionada biblioteca. Luego se revisaron todos los registros recuperados para identificar los relacionados con el programa bajo estudio y separar los relacionados con el doctorado de Humanidades cuyas tesis son asignadas a la misma biblioteca. Esta información fue corroborada con los recursos disponibles en Tercer Ciclo donde se indica las tesis leídas por año académico y el Departamento responsable de la misma.

Para realizar los siguientes pasos de este trabajo, se utilizaron diferentes fuentes de información como: las bases de datos Picasso y Teseo, el catálogo de biblioteca de la UC3M, informes publicados y proporcionados por la Oficina de Tercer Ciclo de la UC3M, así como un cuestionario.

- Picasso nos dio acceso a datos estadísticos de la UC3M. La información de dicha base de datos nos permitió conocer la cantidad de estudiantes matriculados por año académico.

- Del catálogo de la biblioteca obtuvimos la clasificación temática de las tesis defendidas, clasificación que fue comparada con la provista por la base de datos Teseo.

- De la Oficina de Tercer Ciclo, conseguimos información actualizada sobre sus fechas de presentación, directores, miembros del tribunal y la procedencia de dichos miembros, pues aunque Teseo también proporciona esta información, no se actualiza con la periodicidad que lo hacen los propios departamentos universitarios. Por otro lado, el personal de dicha Oficina nos proporcionó información de las nacionalidades de procedencia de los estudiantes, si bien en todo momento se respetó la identidad de los matriculados ya que no teníamos acceso a datos personales.

- Con objeto de conocer el perfil actual de los autores de las tesis como investigadores, y el estado en que se encuentra la investigación comenzada con ellas, se redactó y distribuyó un cuestionario a los autores de las mismas. Al momento de comenzar esta investigación aun no estaban disponibles los datos de las tesis de los años académicos 2006-2007 y 2007-2008, por tal motivo en el cuestionario solo participaron los autores de las tesis defendidas desde comienzos del doctorado hasta el ańo académico 20052006. El mencionado cuestionario nos permitió saber a qué se han dedicado estos autores una vez obtenido el título de doctor, y si la investigación realizada terminó con la defensa de sus tesis o por el contrario ha ido evolucionando. De igual forma se obtuvo información sobre las preferencias de publicación para dar a conocer los resultados obtenidos en sus investigaciones.

\section{Resultados y discusión}

Desde los inicios del programa de Doctorado en 1994 hasta el año académico 2007-2008 se han realizado 789 matriculaciones (no estudiantes sino matrículas). El primer ańo del doctorado es el que mostró menor cantidad de estudiantes matriculados, mientras que los años 2004-2005 y 2005-2006 son los de mayor concentración de alumnos. Desde el año académico 1994-1995 hasta el curso 2007-2008 (14 años) 
se han defendido 58 tesis, un promedio de 4,14 tesis por ańo. Por otra parte, en la misma universidad se han defendido 696 tesis con lo cual el Departamento de Biblioteconomía y Documentación ha defendido el 8,3\% de las tesis defendida en el resto de los departamentos de la UC3M.

Los datos sobre la evolución de la producción de tesis en el periodo estudiado, se representan en la Figura 1.

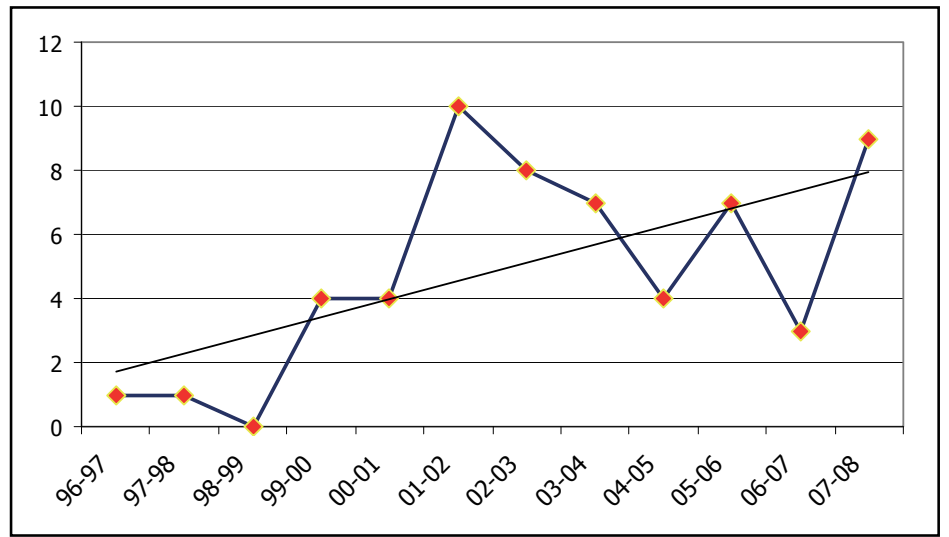

Fig. 1 - Evolución de la producción de tesis en el Departamento de Biblioteconomía y Documentación de la UC3M

Como puede apreciarse, la primera tesis de este Programa Oficial de Postgrado se leyó en el año académico 1996-1997 aunque el doctorado había comenzado en el 1994. Durante estos años se han observado altibajos en dicha producción aunque en términos generales la misma va en un ligero aumento, siendo el 1998-1999 el año de menor producción y el 2001-2002 el año de mayor producción con 10 tesis defendidas.

El promedio de la producción es de 4,14 tesis por año, un valor que aunque en sí mismo no ofrece muchos detalles, cuando se compara con el de otras universidades, indica que se trata de una producción considerablemente buena. Así, el estudio realizado por López Yepes en 2002, para el periodo 1983-2001, seńala que el Departamento de Biblioteconomía y Documentación de la Universidad Complutense, mostraba una producción de 1,9 tesis en su programa doctoral en documentación. Otro estudio hecho en Turquía, indicaba que el promedio era de 3,7 (Tonta \& Al, 2006), y un tercero realizado en India para el mismo nivel académico señaló que, como media, se podían producir 5,7 tesis doctorales anuales en documentación (Vijayakumar, Padmamma, \& Sampatkumar, 2000).

El estudio de la temática de estas tesis se ha basado en la clasificación ofrecida por la biblioteca de la Universidad Carlos III y la base de datos TESEO. Este sistema de gestión de las tesis presentadas en España, está a cargo del Ministerio de Ciencia e Innovación, y recibe la colaboración tanto de los autores de las tesis como de las universidades donde se presentan las mismas. Los autores de las tesis completan una ficha preliminar y las universidades son responsables de validarlas. Las materias son seleccionadas de un Tesauro basado en la clasificación de la UNESCO. (España, 
2009). Las tesis de Documentación de la Universidad Carlos III tienen tendencia a ser clasificadas en Lingüística, Lingüística aplicada, Documentación y Documentación aplicada, seguidas de Ciencias jurídicas. Se reconoce la importancia de Teseo para las investigaciones basadas en estudios de tesis, sin embargo, como su clasificación temática es muy general, optamos por la clasificación asignada por la biblioteca para realizar nuestro análisis. En el caso de la biblioteca vemos como los temas más tratados en las tesis bajo estudio son: bibliometría y temas relacionados con bibliotecas e investigación científica seguidos de la gestión de documentos y del conocimiento, la documentación tanto general como automatizada y la archivística.

En general, la investigación que se lleva a cabo para realizar la tesis, tiene que estar relacionada con el departamento donde se defiende. Sin embargo, si observamos la relación de estas tesis con temáticas que pueden quedar fuera de las que están directamente relacionadas con la biblioteconomía y documentación, encontramos que el 35,2\% del total de temas identificados no están relacionados con la documentación. Ahora bien, en ese porcentaje hay muchos temas distintos, y cada uno en si mismo tiene una representación baja en comparación con la totalidad de la temática, pues la mayoría no llega al $1 \%$ del total de temas tratados en las tesis.

En la tabla 1 se recogen los temas asignados por la biblioteca que aparecen con un porcentaje superior al 1\%. Entre ellos, destacan los lenguajes de programación e Internet, seguidos de evaluación y ciencias entre otros temas presentados.

Tabla 1 - Representación de temas externos a la documentación que han sido tratados en las tesis del Departamento de Biblioteconomía y Documentación de la UC3M

\begin{tabular}{|l|c|}
\hline Temas externos a la documentación & \% según el total de temas (125) \\
\hline XML, Web, Internet, hipertexto & 7,2 \\
\hline Evaluación & 3,2 \\
\hline Ciencias, Ciencias naturales y científicos & 2,4 \\
\hline Jurisprudencia, Derecho Mercantil, Legislación & 2,4 \\
\hline Análisis comparativo y cuantitativo & 1,6 \\
\hline Fotografía & 1,6 \\
\hline Personas con discapacidad, Discapacidad física & 1,6 \\
\hline
\end{tabular}

Por otro lado cabe señalar que las temáticas más representadas que no pertenecen al área de la biblioteconomía y documentación provienen de ramas temáticas muy afines. Este es el caso de Internet que pese a que algunos tesauros no lo ubican en el área de documentación (UNESCO, 2009), desde hace varios años ha tenido mucha presencia en las investigaciones de esta área. Otro ejemplo es el caso de la Jurisprudencia que aunque no pertenece a la biblioteconomía y documentación está muy relacionado con ésta por pertenecer ambas a la rama de ciencias sociales. De esto se deduce que las tesis leídas en este departamento se relacionan principalmente con temáticas del propio departamento pero también con temáticas afines, y bastante menos con temáticas externas a la profesión como por ejemplo las Ciencias y la Fotografía. Los doctorandos participan más con disciplinas afines a la documentación y es muy escasa la participación con disciplinas menos relacionadas. Esto confirma los resultados obtenidos en un estudio donde mediante la clasificación de revistas se buscaba conocer la relación entre 
disciplinas encontrando que las Ciencias sociales y las humanidades se relacionan mucho con disciplinas próximas y muy poco externamente (Morillo, 2000).

Nuestro segundo objetivo trataba de identificar la visibilidad del Departamento de Biblioteconomía y Documentación mediante la participación de instituciones extranjeras en los procesos de producción y evaluación de las tesis. La visibilidad de un departamento puede observarse con diferentes alternativas. Tenemos el caso de estudios que la observan mediante las redes sociales (Perianes-Rodríguez, 2008), otros mediante la producción en bases de datos internacionales (Iribarren, 2006; Pinto, 2007) y otros desde bases de datos nacionales (Suárez, 2004).

Para conseguir dicho objetivo, se ha estudiado como se refleja esa visibilidad en la capacidad para atraer investigadores que participan tanto en la co-dirección de las tesis como en los tribunales de las mismas. Al mismo tiempo se observa la capacidad para atraer futuros doctores de otras nacionalidades, a que se formen académicamente en la Universidad Carlos III. Para estudiar este aspecto, hemos considerado las aportaciones internacionales que recibe el Departamento de Biblioteconomía y Documentación mediante la participación de investigadores pertenecientes a instituciones extranjeras tanto en los comités de tesis como en la co-dirección de las mismas. También se analiza la procedencia de los alumnos matriculados.

En la tabla 2 se recogen las instituciones de las que proceden los doctores que participaron tanto en la producción como en la evaluación de las tesis, así como el número de firmas que han aportado.

Tabla 2 - Aportación de instituciones extranjeras a las tesis presentadas en el Departamento de Biblioteconomía y Documentación en la UC3M

\begin{tabular}{|l|c|c|c|}
\hline Institución & $\begin{array}{c}\text { Firmas } \\
\text { aportadas }\end{array}$ & $\begin{array}{c}\% \text { sobre } \\
\text { el total de } \\
\text { firmas (370) }\end{array}$ & $\begin{array}{c}\text { \% sobre } \\
\text { firmas } \\
\text { externas } \\
(174)\end{array}$ \\
\hline U de Brasilia (Brasil) & 4 & 1,08 & 2,30 \\
\hline U de Sao Paulo (Brasil) & 4 & 1,08 & 2,30 \\
\hline Biblioteca Nacional de Portugal (Portugal) & 1 & 0,27 & 0,57 \\
\hline Centre for science and technologies studies (Francia) & 1 & 0,27 & 0,57 \\
\hline INIST (Francia) & 1 & 0,27 & 0,57 \\
\hline U de Leiden (Holanda) & 1 & 0,27 & 0,57 \\
\hline U de Puerto Rico (Puerto Rico) & 1 & 0,27 & 0,57 \\
\hline U de Rennes 2 (Francia) & 1 & 0,27 & 0,57 \\
\hline U Federal Flumense de Río de Janeiro (Brasil) & 1 & 0,27 & 0,57 \\
\hline U Nacional de México (México) & 1 & 0,27 & 0,57 \\
\hline U Oporto de Portugal (Portugal) & 1 & 0,27 & 0,57 \\
\hline U Pontificia Católica de Campińas Brasil (Brasil) & 1 & 0,27 & 0,57 \\
\hline U Stendhal Grenoble III (Francia) & 1 & 0,27 & 0,57 \\
\hline U Técnica de Lisboa (Portugal) & 1 & 0,27 & 0,57 \\
\hline U Wolverhampton (Inglaterra) & 1 & 0,27 & 0,57 \\
\hline U de Aix-Marseille III CRRM (Francia) & 1 & 0,27 & 0,57 \\
\hline
\end{tabular}


En las 58 tesis leídas en el periodo bajo estudio, han participado 98 profesores externos a la Universidad Carlos III actuando tanto de co-directores de tesis como de miembros de tribunales. Dichos profesores provienen tanto de instituciones españolas como extranjeras. La procedencia de estas instituciones extranjeras son: Brasil, Francia, Portugal, Holanda, Inglaterra, México y Puerto Rico. Estas instituciones extranjeras han aportado 174 firmas del total de 370 firmas, siendo Brasil es el país que más firmas aporta como puede verse en la tabla 2 .

Por otra parte, se ha observado la procedencia internacional de los alumnos matriculados. De acuerdo a los datos suministrados por la Oficina de Tercer Ciclo de la UC3M, por razones obvias, la procedencia principal es España, mientras la procedencia de estudiantes internacionales recae entre otros, sobre los siguientes países: Brasil, Chile, Colombia, Estados Unidos, México, Panamá, Puerto Rico y Venezuela. Ello implica que la Universidad Carlos III de alguna forma ha tenido presencia en dichos países, hasta el punto de que ha conseguido atraer estudiantes a su programa de Doctorado en Documentación. Sin embargo, no se percibe que haya tenido el mismo efecto en Europa puesto que sus alumnos internacionales en Documentación provienen principalmente de América. Brasil, México y Puerto Rico destacan entre el resto, ya que de estos países no sólo se han formado doctores, sino que también han aportado doctores para participar en las tesis.

Tanto la visibilidad como la colaboración internacional han tenido repercusión en la investigación para otros países ya que el $25 \%$ de las tesis guarda relación con la realidad de países no españoles como: Brasil, Latinoamérica y el Caribe, Puerto Rico, Europa, México, Países que componen MERCOSUR y Países Lusófonos. Aunque somos conscientes de que Brasil pertenece a los Países Lusófonos, Puerto Rico al Caribe y México a Latinoamérica, se prefirió conservar la estructura establecida por el autor de la tesis. Entendemos que una tesis sobre Puerto Rico, por ejemplo, puede estar centrada en la realidad de ese país sin que implique que se considera la situación de otros países que componen el Caribe. De igual forma, una tesis que observa la situación de Latinoamérica y el Caribe implica un área mayor. Siguiendo con esta misma línea de pensamiento, las tesis relacionadas con la situación de España no están contempladas dentro de la categoría internacional Europa. Esta visibilidad, observada en la temática de las tesis, ha permitido a la Universidad Carlos III no solamente darse a conocer en otras partes del mundo sino colaborar y aportar conocimientos y recursos a la investigación que se lleva a cabo en otros países, principalmente en América. El estudio de la relación entre el área geográfica donde se realiza la investigación y el tema de la tesis, ha permitido elaborar la tabla 3.

Tabla 3 - Relación entre los países donde se realiza la investigación y los temas tratados

\begin{tabular}{|l|l|}
\hline País & Temas \\
\hline Brasil & Bibliotecas; Sociedad de la Información; Bibliometría \\
\hline Europa & Documentación; Web; Análisis cuantitativo \\
\hline Latinoamérica y Caribe & $\begin{array}{l}\text { Investigación científica; Bibliometría; Biblioteconomía; Archivística; } \\
\text { Personas con discapacidad }\end{array}$ \\
\hline
\end{tabular}




\begin{tabular}{|l|l|}
\hline México & $\begin{array}{l}\text { Archivística; Gestión de documentos; Investigación científica; Bibliometría; } \\
\text { Agricultura }\end{array}$ \\
\hline Mercosur & Políticas de información \\
\hline Puerto Rico & Bibliotecas; Bibliometría; Ciencias; Archivística \\
\hline
\end{tabular}

En dicho estudio se encontró que las investigaciones de tesis realizadas en Brasil y presentadas en la UC3M están relacionadas con bibliotecas, sociedad de la información y bibliometría. Cuando se trataba de Europa, se trabajaba sobre temas de documentación en general, web y análisis cuantitativo. En las tesis realizadas con investigaciones en Latinoamérica y el Caribe se han tratado los temas de investigación científica, bibliometría, biblioteconomía, archivística y personas con discapacidad. Por lo que respecta a México, se ha investigado en temas de archivística, gestión de documentos, investigación científica, bibliometría y documentación sobre agricultura. Los países que componen el MERCOSU han sido estudiados en base a políticas de información, mientras el caso de Puerto Rico ha sido investigado con temas de bibliotecas, bibliometría, ciencias y archivística.

Esta clasificación temática por países es de gran utilidad para personas interesadas en comenzar estudios doctorales en documentación ya que les permitiría ver la relación de la Universidad Carlos III con su país de origen. Por ejemplo, un estudiante de Brasil interesado en temas de sociedad de la información sabe que en esta universidad existen unas relaciones previas donde investigadores de la misma conocen la realidad social de su país lo cual es de gran ayuda al momento de seleccionar la universidad y comenzar la investigación conducente al doctorado.

Como tercer objetivo nos proponíamos conocer el perfil actual de los Doctores que se formaron como investigadores en el Departamento de Biblioteconomía y Documentación de la Universidad Carlos III de Madrid, y además, si estos doctores dieron continuidad a la investigación que iniciaron en su tema de tesis. Para conseguirlo, se envió un cuestionario a los autores de las tesis defendidas desde el comienzo del doctorado hasta el año académico 2005-2006. Al momento de comenzar esta investigación y distribuir el cuestionario no estaban disponibles la totalidad de las tesis defendidas en los años académicos 2006-2007 y 2007-2008 por este motivo no se incluyeron en esta sección de la investigación.

Los participantes en el estudio se identificaron principalmente como profesores, seguidos de los bibliotecarios y luego los documentalistas. Debido a que la mayoría son profesores no sorprende que el principal centro de trabajo sean las instituciones educativas. Este hallazgo confirma lo publicado por el Instituto Nacional de Estadística (2008), que indica que la mayoría de los doctores españoles trabajan en el sector de enseñanza superior. En España esta afirmación se apoya en que para acceder a puestos de profesor permanente en la universidad, obligatoriamente se tiene que poseer el título de Doctor. Para el $83 \%$ de los encuestados la investigación es parte de sus tareas principales en su trabajo.

La mayoría de los encuestados continúa trabajando en su tema de tesis y lo han ido enriqueciendo con temas nuevos, sin embargo, el 28,6\% de ellos ha descartado su tema de tesis y tiene una línea de investigación distinta. Es interesante ver como la archivística es uno de los temas más investigados a pesar de que sus autores no trabajan en archivos. 
Esto se explica con un estudio publicado por Recorder y Cid (1998) que indica que los profesionales de un sector escriben menos que los doctores y/o investigadores.

Con relación a las preferencias de publicación siempre son las revistas la primera opción. Cuando se trata de publicar los resultados de sus tesis escogen revistas y libros, sin embargo, cuando presentan los resultados de otras investigaciones diferentes a la tesis doctoral prefieren revistas y congresos. De igual forma para mantenerse informados de los avances en sus temas de investigación utilizan revistas, sin embargo los libros y los congresos tienen la misma aceptación (23\% cada uno).

\section{Conclusiones}

El departamento de Biblioteconomía y Documentación de la Universidad Carlos III produce una media de 4,14 tesis anuales. Las tesis bajo estudio representan el 8,3\% del total de tesis que produce esta universidad para el mismo periodo de tiempo. Dicha cantidad es saludable considerando que esta universidad cuenta con 18 programas de doctorado en el periodo estudiado. De igual forma, este resultado es similar a la producción de otros departamentos de documentación en otras universidades tanto en España como en otros países.

Por lo que respecta a la temática de las tesis, están centradas en temas de documentación por razones evidentes. Sin embargo, se observó que el 35,2\% de los temas no son de biblioteconomía y documentación. Los temas externos a la Biblioteconomía y Documentación son varios, pero en general con poca frecuencia ya que la mayoría no representa el $1 \%$ del total de temas.

Por otra parte, en lo que respecta a la visibilidad del departamento en función de los estudiantes extranjeros matriculados, se percibe mayor aceptación en América que en Europa. Sin embargo, cuando se hace en función de la colaboración en las tesis mediante co-dirección o participando en los tribunales indica una participación equitativa entre América y Europa. Se entiende que al departamento en su relación con Europa le resulta más fácil atraer doctores que doctorandos, sin embargo con América la situación es diferente, pues además de recibir colaboraciones de otros doctores también forma nuevos doctores.

Por lo que respecta al perfil actual de los doctores formados en este departamento, estos mayoritariamente continúan con su tema de tesis y solo cerca del $29 \%$ ha abandonado la investigación que comenzó con la tesis. Dichos doctores se ubican principalmente en tareas docentes. Considerando que la investigación es lo que permite a una profesión evaluarse y evolucionar, y que los principales investigadores que publican el resultado de sus investigaciones están en la Universidad, debemos pensar que el desarrollo de la biblioteconomía y documentación está íntimamente ligado al ambiente académico. Sin embargo, los profesionales del sector viven de primera instancia los cambios y necesidades de la profesión aunque estudios anteriores revelan que estos publican menos. Un caso interesante es el de los autores de tesis con temas de archivística que al momento de la encuesta no trabajan en archivos aunque continúan investigando sobre el tema. 


\section{Referências bibliográficas}

España, Ministerio de Ciencias e Innovación. TESEO. Recuperado el 15 de mayo de 2009 de https://www.micinn.es/teseo/ayuda/MU\%20TESEO4.htm

Instituto Nacional de Estadística. (2008). Encuesta sobre recursos humanos en Ciencia y Tecnología 2006. Resultados provisionales. Recuperado el 15 de mayo de 2009 de http://www.ine. es/prensa/np509.pdf.

Iribarren Maestro, I. (2006). Producción cientifica y visibilidad de los investigadores de la Universidad Carlos III de Madrid en las Bases de Datos del ISI, 1997-2003. Tesis doctoral, Universidad Carlos III de Madrid.

López Yepes, J. (2002). Focos de investigación y escuelas científicas en documentación a través de la realización y dirección de tesis doctorales. El caso del Departamento de Biblioteconomía y Documentación de la Universidad Complutense de Madrid (1983-2001). Documentación de las Ciencias de la Información. Vol. 25: 19-54.

Recuperado el 10 de mayo de 2009, de http://revistas.ucm.es/inf/02104210/articulos/ DCIN0202110019A.PDF

Morillo Ariza, F. (2000). Estudio de la Interdisciplinariedad en la ciencia a través de indicadores bibliométricos. Tesis doctoral, Universidad Carlos III de Madrid.

Perianes-Rodríguez, A. (2008). Análisis y visualización de redes de colaboración científica. Grupos de investigación en la Universidad Carlos III (ISI, Web of Science, 1990-2004). Tesis doctoral, Universidad Carlos III de Madrid.

Pinto, AL. (2007). Análisis de la producción cientifica en bibliotecnomía y documentación en el periodo 1995/204: estudio en los principales programas españoles y brasileños. Tesis doctoral, Universidad Carlos III de Madrid.

Recoder Sellares, MJ \& Cid Leal, Pilar. (1998). Los intereses profesionales de bibliotecarios y documentalistas en España a través de su participación en las Jornadas Españolas de Documentación Automatizada: doce años de ponencias, comunicaciones y experiencias. FESABID 98: VI Jornadas Españolas de Documentación. Recuperado el 10 de mayo de 2009 de http://fesabid98.florida-uni.es/Comunicaciones/p_cid.htm.

Sanz Casado, E. \& Martín Moreno, C. (1997). Técnicas bibliométricas aplicadas a los estudios de usuarios. Revista General de Información y Documentación. Vol. 7(2): 41-68.

Suárez Balseiro, CA. (2004). Perfiles de actividad cientifica de los departamentos de la Universidad Carlos III de Madrid: un estudio con variables de recursos y resultados del proceso científico durante el periodo de 1998 a 2001. Tesis doctoral, Universidad Carlos III de Madrid.

Tonta, Y. \& Al, U. (2006). Scatter and obsolescence of journals cited in theses and dissertations of librarianship. Library \& Informations Sciences Research. Vol. 28(2): 281-296.

Universidad Carlos III, Departamento de Biblioteconomía y Documentación. (2009). Historia. Recuperado el 5 de mayo de 2009 de http://www.uc3m.es/portal/page/portal/ biblioteconomia_documentacion/conozcanos/historia

UNESCO. Thesauro de la UNESCO. Recuperado el 15 de mayo de 2009 de http://databases. unesco.org/thessp/

Vijayakumar, M., Padmamma, S \& Sampatkumar, BT. (2000). Information use by library professionals: a scientometric analysis. ILA Bulletin Vol. 36 (1): 19-23. Recuperado el 1 de mayo de 2009 de http://eprints.rclis.org/3312/1/pdf.pdf 